\title{
Brief Communication: The double layer in the separatrix region during magnetic reconnection
}

\author{
J. Guo ${ }^{1,2}$ and B. Yu ${ }^{1}$ \\ ${ }^{1}$ School of Mathematics and Physics, Qingdao University of Science and Technology, Qingdao, Shandong 266061, China \\ ${ }^{2}$ Sate Key Laboratory of Space Weather, Chinese Academy of Sciences, Beijing 100190, China \\ Correspondence to: J. Guo (guojun@qust.edu.cn, guoj2005@163.com)
}

Received: 3 October 2014 - Published in Nonlin. Processes Geophys. Discuss.: 5 November 2014

Revised: 10 February 2015 - Accepted: 1 March 2015 - Published: 16 March 2015

\begin{abstract}
With two-dimensional (2-D) particle-in-cell (PIC) simulations we investigate the evolution of the double layer (DL) driven by magnetic reconnection. Our results show that an electron beam can be generated in the separatrix region as magnetic reconnection proceeds. This electron beam could trigger the ion-acoustic instability; as a result, a DL accompanied with electron holes (EHs) can be found during the nonlinear evolution stage of this instability. The spatial size of the DL is about 10 Debye lengths. This DL propagates along the magnetic field at a velocity of about the ion-acoustic speed, which is consistent with the observation results.
\end{abstract}

\section{Introduction}

Magnetic reconnection is a very important process in space plasma, which can convert the magnetic field energy into kinetic energy of electrons and ions (Pritchett, 2006; Lu et al., 2010). In previous work, more attention is paid to the whistler waves (Fujimoto and Sydora, 2008; Deng and Matsumoto, 2001; Guo, 2011; Xiao et al., 2007, 2008, 2010) and electron accelerations (Fu et al., 2006; Huang et al., 2010; Pritchett, 2006). Recently, the electrostatic waves have been found in the simulations and observations. With the particlein-cell (PIC) simulation, Drake et al. (2003) reported that the electrostatic waves and electron holes (EHs) are produced by the Buneman instability. In their simulation, the initial electron drift speed is above the threshold to trigger the Buneman instability, except for a strong guide field. However, Fujimoto and Machida (2006) suggested that the electrostatic waves are excited by the electron two-stream instability which has a relationship with magnetic reconnection. Particularly, no guide field is used in their initial condition. The observation (Li et al., 2009) proved that the guide field has no direct effect on the generation of the electrostatic wave in or around the reconnection diffusion region. These reported electrostatic waves appear with bipolar electric field and EHs, while the signature of the double layer (DL) is a unipolar electric field. The electrons and ions will be accelerated, decelerated, or reflected by the electric field when they enter the DL. The current-driven DLs have been carefully studied by using a series of one-dimensional (1-D) Vlasov simulations (Singh, 1980, 1982, 2000). It is found that EHs are a common feature of a strong DL. The evolution of the electron beam accelerated by a DL would contribute to continuously creating moving EHs. Additionally, the DL can accelerate the ion beam which can generate fluctuations above the ion cyclotron frequency in the 2.5-D PIC simulations (Singh and Khazanov, 2003).

Electron holes are frequently observed by the spacecraft observation during magnetic reconnection. Double layers are frequently observed in the auroral ionosphere (Boström, 1992; Ergun et al., 2001). Only recently, Ergun et al. (2009) reported DLs in the plasma sheet in the magnetotail, but they could not confirm the relation between the DL and magnetic reconnection in their work. Wang et al. (2014) presented the first evidence of DLs during magnetic reconnection, and found that DLs are moving away from X line at a velocity of about ion-acoustic speed in the separatrix region. Meanwhile, a series of EHs moving toward the $\mathrm{X}$ line are observed in the wake of the DL. In addition, the multiple DLs are also observed in the simulations (Singh et al., 2011).

The electrostatic waves have a close relationship with electron beams. Previous simulations show that electron beams 

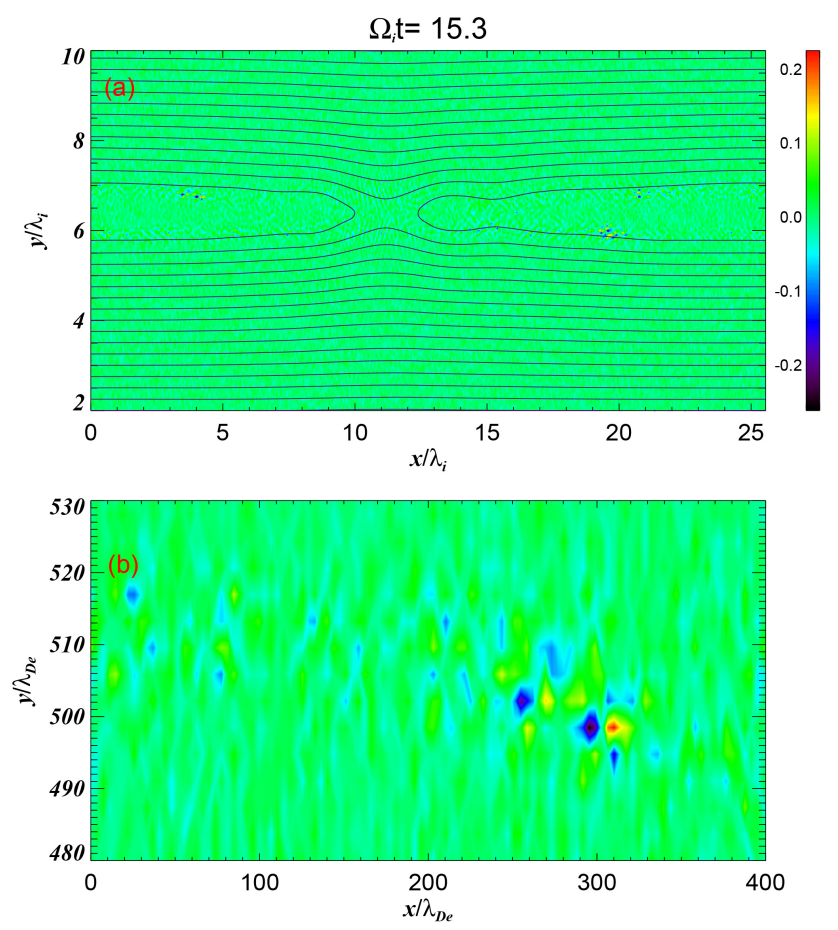

Figure 1. The electric field parallel to the local magnetic filed $E_{/ /}$ and magnetic field lines at $t \Omega_{\mathrm{i}}=15.3$ (a), the magnification of the left-hand plane (b).

form mainly close to the separatrix (Hoshino et al., 2001). Using 3-D simulation in the presence of a guide field, Pritchett and Coroniti (2004) found the enhanced parallel electric field and electron velocity are confined to one pair of separatrix. In this case strong DLs can be generated at the reconnection site. The purpose of our study is to investigate the generation mechanism of the DL during the magnetic reconnection without a guide field. Our results suggest that both the DL and EH can be formed in our simulation. These polarized structures should be excited by the ion-acoustic instabilities.

\section{Simulation model}

The initial magnetic field distribution is set to be $B_{x}(y)=$ $B_{0} \tanh \left[\left(y-L_{y} / 2\right) / \delta_{0}\right]$, where $\delta_{0}$ is the half width of the initial current sheet. The particle densities read $n(y)=$ $n_{0} \operatorname{sech}^{2}\left[\left(y-L_{y} / 2\right) / \delta_{0}\right]+n_{\mathrm{b}}$, where $n_{0}$ and $n_{\mathrm{b}}$ represent the current sheet and background densities. $\delta_{0}$ is equal to $0.5 \lambda_{\mathrm{i}}$, where $\lambda_{\mathrm{i}}$ is the ion inertial length given by $n_{0}$. All of the particles have the initial Maxwellian velocity distributions. In the present simulation, the simulation size is $L_{x} \times L_{y}=25.6$ $\lambda_{\mathrm{i}} \times 12.8 \lambda_{\mathrm{i}}$. The other parameters are $T_{\mathrm{i}} / T_{\mathrm{e}}=5, n_{\mathrm{b}}=0.1 n_{0}$, $m_{\mathrm{i}} / m_{\mathrm{e}}=256$, and $c / V_{\mathrm{A}}=30$, where $V_{\mathrm{A}}$ is the Alfvén speed based on $B_{0}$ and $n_{0}$; the ratio of ion gyrofrequency to plasma frequency is $\omega_{\mathrm{pi}} / \Omega_{\mathrm{i}}=c / V_{\mathrm{A}}=30$. The spatial resolution is $\Delta x=\Delta y=0.05 \lambda_{\mathrm{i}}$. The time step is $\Delta t \Omega_{\mathrm{i}}=0.001$, where
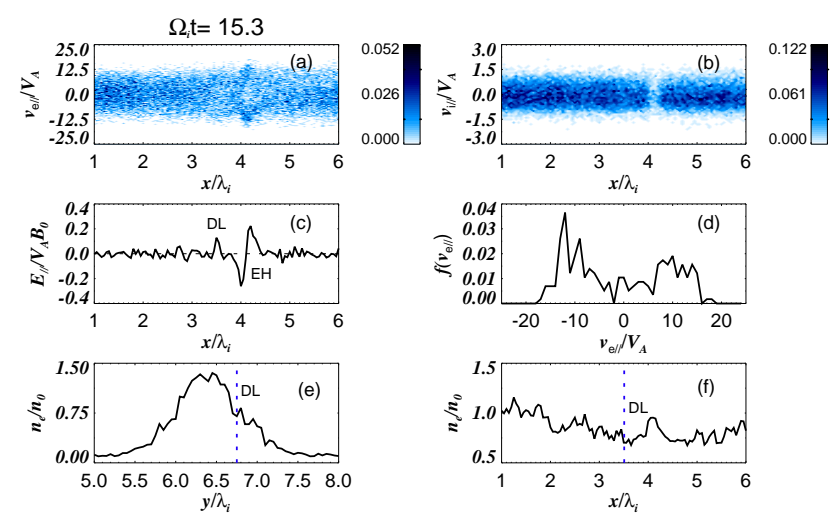

Figure 2. The $v_{/ /}-x$ phase-space distributions of electron (a) and ion (b), and the electric field $E_{/ /}$at $y / \lambda_{\mathrm{i}}=6.75(\mathbf{c})$, the parallel velocity distribution of electron $f\left(v_{\mathrm{e} / /}\right)$ at about $x / \lambda_{\mathrm{i}}=4.1$ and $y / \lambda_{\mathrm{i}}=6.75(\mathbf{d})$, the density distribution of electron along $y$ axis at $y / \lambda_{i}=3.5$ where the double layer (DL) appear (e) and the density distribution of electron along $x$ axis at $y / \lambda_{\mathrm{i}}=6.75$ (f).

$\Omega_{\mathrm{i}}$ is the proton cyclotron frequency. Along the $x$ axis, the periodic boundary conditions are used, and the ideal conducting boundary conditions are used in the $y$ axis. The particles will be reflected when they reach the boundary in the $y$ axis. The magnetic and electric fields are calculated with a full explicit algorithm. About $6 \times 10^{6}$ particles per species are employed in the simulation. A small initial magnetic perturbation is superposed in the form of which is the same as in the references (Lu et al., 2013; Guo, 2014).

\section{Simulation results}

Figure 1a shows the electric field parallel to the local magnetic field $E_{/ /}$and magnetic field lines at $t \Omega_{\mathrm{i}}=15.3$. Figure $1 \mathrm{~b}$ shows the magnification of $E_{/ /}$in left-hand plane. We plot Fig. $1 \mathrm{~b}$ as a function of the electron Debye length $\lambda_{\mathrm{De}}$ instead of the ion inertial length; $\lambda_{\mathrm{De}}=v_{\text {the }} / \omega_{\text {pe }}$ is the initial electron Debye length. At this time, the electron thermal speed is about $v_{\text {the }} \sim v_{\text {th } 0}=\left(2 T_{\mathrm{e}} / m_{\mathrm{e}}\right)^{1 / 2} \sim 6.5 V_{\mathrm{A}}$. It is obvious that electric fields with polarized structures are mainly located in the separatrix region. These structures are almost along the magnetic field lines. As the magnetic reconnection proceeds, the polarized electric fields will also appear in the diffusion region, which have been discussed in our previous work (Guo, 2014).

Shown in Fig. 2 are the $v_{/ /}-x$ phase-space distributions of electron (a) and ion (b), and the electric field $E_{/ /}$at $y / \lambda_{\mathrm{i}}=6.75$ (c). Figure $2 \mathrm{a}$ and c show clearly that there is an electron phase-space hole at $x / \lambda_{\mathrm{i}} \sim 4.1$. Besides that, a DL with a width about $10 \lambda_{\text {De }}$ lies at $x / \lambda_{\mathrm{i}} \sim 3.5$, which is in the wake of a EH. Figure $2 \mathrm{~d}$ is the parallel velocity distribution of electron $f\left(v_{\mathrm{e} / /}\right)$ at about $x / \lambda_{\mathrm{i}}=4.1$ and $y / \lambda_{\mathrm{i}}=6.75$, which corresponds to the EH shown in Fig. 2a. This distribution appears an electron beam with $v_{\mathrm{e} / / /} / V_{\mathrm{A}} \sim-12.0$ and a 

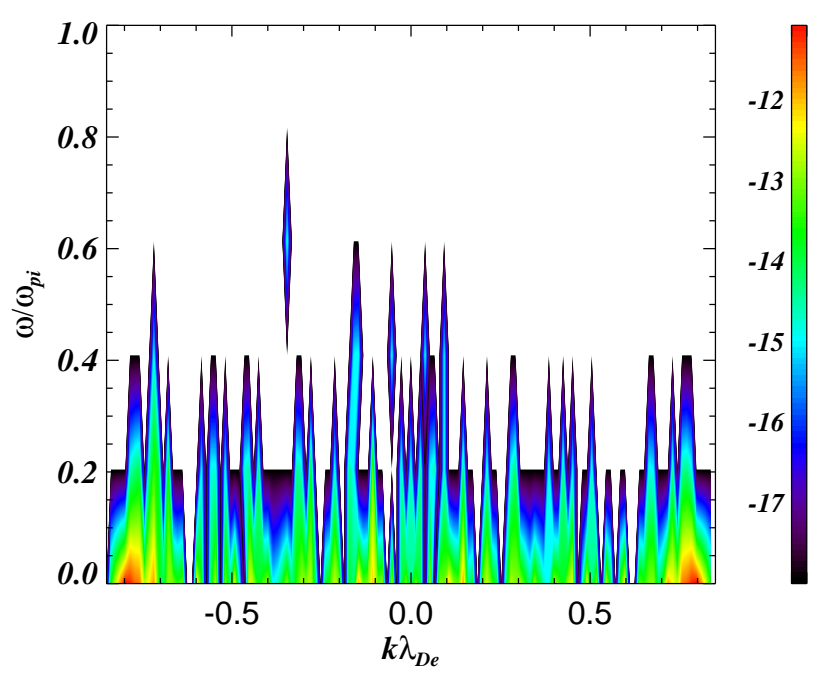

Figure 3. The wave spectrum of parallel electric field. The sampling region is $0<x / \lambda_{\mathrm{i}}<6.4$ and the time interval is $15.0<t \Omega_{\mathrm{i}}<15.5$.

broad peak centered around $v_{\mathrm{e} / /} / V_{\mathrm{A}} \sim 10.0$ (the flat-top distribution). On the other hand, the low-velocity portion of the distribution is also dominated by a plateau formation. Figure $2 \mathrm{e}$ is the density distribution of electron along $y$ axis at $y / \lambda_{\mathrm{i}}=3.5$ where the DL appears. Figure $2 \mathrm{f}$ is the density distribution of electron along $x$ axis at $y / \lambda_{\mathrm{i}}=6.75$. By coupling Fig. 2e and $\mathrm{f}$, it is found that the density of electrons where the DL appears is lower than the surrounding, along both $x$ and $y$ axes. Based on the observations (Wang et al., 2013, 2014), the DL and EH are observed together. Moreover, the structure of DLs in our simulation is basically consistent with the observation results.

The magnetic field line passes through $y / \lambda_{\mathrm{i}}=6.75$, where the magnetic field is nearly parallel to the $x$ axis in the region $x / \lambda_{\mathrm{i}}<7$. The plateau function means that the Buneman or ion-acoustic modes probably appear. Figure 3 is the wave spectrum of a parallel electric field. The sampling region is $0<x / \lambda_{\mathrm{i}}<6.4$ and the time interval is $15.0<t \Omega_{\mathrm{i}}<15.5$. It is found that the normalized wave number associated with the highest wave energy density is $k \lambda_{\mathrm{De}} \sim \pm 0.8$. These forward and backward modes correspond to the distribution shown in Fig. 2d. During this period, the frequency of the dominant mode is far below the ion plasma frequency. All of the above suggests that the DL located in the separatrix region shown in Fig. 2c should be produced by the ion-acoustic instabilities.

Both numerical simulations and observations have been performed during the past decades which show that DLs are highly variable structures moving with time (Singh et al., 2005; Wang et al., 2014). Figure 4a is the time evolution of the polarized electric field shown in Fig. $1 \mathrm{~b}$ at $y / \lambda_{\mathrm{i}}=6.75$. The DL in our simulation seems to only propagate outward along the magnetic field for a short distance. The propagation velocity can be estimated to be $0.2 V_{\mathrm{A}}$, while the DL found in the separatrix near the $X$ line shown in Fig. $4 \mathrm{~b}$ can propagate
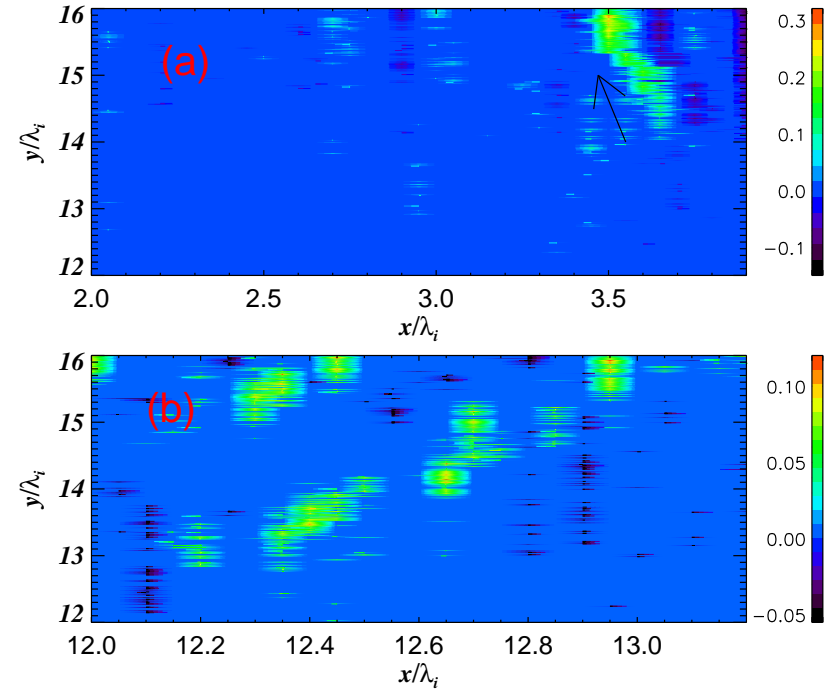

Figure 4. The time evolution of the DL shown in Fig. $1 \mathrm{~b}$ at $y / \lambda_{\mathrm{i}}=$ 6.75 (a) and the DL found in the separatrix near the $\mathrm{X}$ line (b).

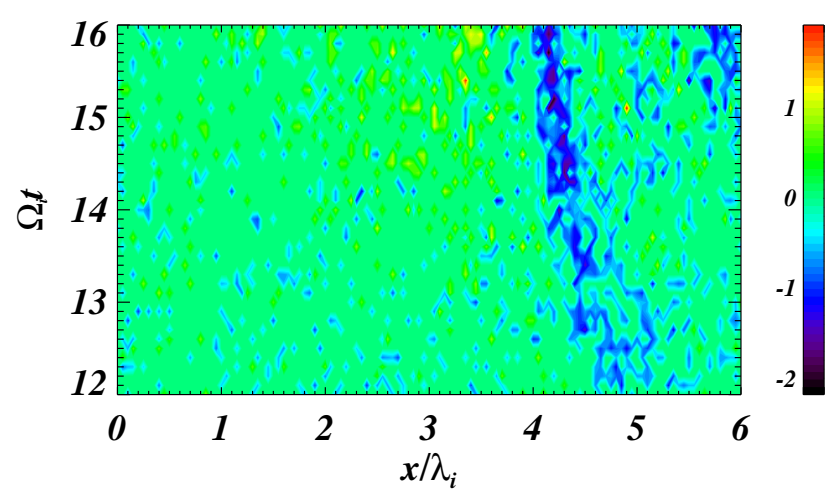

Figure 5. The time evolution of the electron flow velocity parallel to the magnetic field at $y / \lambda_{i}=6.75$.

for a long time and distance. The propagation velocity can be estimated to be $0.4 V_{\mathrm{A}}$. In fact, these two velocities are on the same order of the ion-acoustic speed, although the former is a little small. The observation (Wang et al., 2014) also suggests that the DL propagates away from the $\mathrm{X}$ line at a velocity of about ion-acoustic speed; that is, the results shown in Fig. 4 are very consistent with those observations. However, the DL found in the separatrix at a distance of several ion inertial lengths away from the $\mathrm{X}$ line shown in Fig. $1 \mathrm{~b}$ cannot propagate for a long distance. A reasonable explanation for Fig. 4a is due to the periodic boundary condition.

Previous simulation results about magnetic reconnection (Hoshino et al., 2001; Pritchett and Coroniti, 2004) show that electron beams form mainly close to the separatrix. In this condition, strong DLs can be generated at the reconnection site. These simulation results have been confirmed by the cluster observations published by Vaivads et al. (2004). 
Figure 5 shows the time evolution of the electron flow velocity parallel to the magnetic field at $y / \lambda_{i}=6.75$. The localized electron beam with negative drift velocity propagates along the magnetic field toward the left. At time $t \Omega_{\mathrm{i}} \sim 12$, this electron beam lies at $x / \lambda_{\mathrm{i}} \sim 4.8$, while the propagation stops at $x / \lambda_{\mathrm{i}} \sim 4.1$ due to the periodic boundary condition at time $t \Omega_{\mathrm{i}} \sim 15.5$. This evolution is consistent with that of the parallel electric field shown in Fig. 4a. Of course, the DL appears after the electron beam formed for a period of time. On the other hand, as the time goes on, the width of electron flow becomes smaller, while the beam velocity becomes larger. The figure shows that only when the velocity of electron beam reaches a certain value the electrostatic waves can be excited. Compared with Fig. 2, the electron beam is located in the region where the EH appears. Our previous simulation results (Guo and $\mathrm{Li}, 2013$ ) suggest that the ion-acoustic waves are always accompanied by electron beams. Together with Fig. 2d, it can be thus concluded that the localized electron beam excites the ion-acoustic instabilities, which results in the formation of DL. However, the electron beam cannot drift for a long time due to the periodic boundary, which leads the DL to not propagate over a long distance.

\section{Conclusions}

In this paper, the evolution of DLs during magnetic reconnection is studied by 2-D electromagnetic PIC simulation. The simulation results show that the DLs can be found in the separatrix region, which are generated by ion-acoustic instability. The DL found in our simulation is about 10 Debye lengths and propagates away from the diffusion region with a velocity of about the ion-acoustic speed. These results are consistent with the observation results (Wang et al., 2014). The physical evolution process can be inferred from the following processes. At first, the electrons are accelerated near the $\mathrm{X}$ line and then flow out of the diffusion region along the magnetic field. When the velocity of the electron beam is large enough, the localized electron beam excites the ionacoustic instabilities, which produced the DL. But the DLs at a few ion inertial lengths away from the $\mathrm{X}$ line can only propagate for a short distance, because the propagation of electron beam tends to be stable due to periodic boundary conditions. However, even though we get some interesting results, there are still many questions that remain to be addressed. For instance, a sufficiently long system will be used to study the propagation of DLs in future work.
Acknowledgements. This research was supported by the National Natural Science Foundation of China under grant no. 41204115 and the Specialized Research Fund for State Key Laboratories.

Edited by: B. Tsurutani

Reviewed by: N. Singh and one anonymous referee

\section{References}

Boström, R.: Obdervations of weak double-layers on auroral field lines, IEEE Trans. Plasma Sci., 20, 756-763, 1992.

Deng, X. H. and Matsumoto, H.: Rapid magnetic reconnection in the Earth's magnetosphere mediated by whistler waves, Nature, 410, 557-560, 2001.

Drake, J. F., Swisdak, M., Cattell, C., Shay, M. A., Rogers, B. N., and Zeiler, A.: Formation of electron holes and particle energization during magnetic reconnection, Science, 299, 873-877, 2003.

Ergun, R. E., Su, Y. -J., Andersson, L., Carlson, C. W., McFadden, J. P., Mozer, F. S., Newman, D. L., and Goldman, M. V.: Direct observation of localized parallel electric fields in a space plasma, Phys. Rev. Lett., 87, 045003, doi:10.1103/PhysRevLett.87.045003, 2001.

Ergun, R. E., Andersson, L., Tao, J., Angelopoulos, V., Bonnell, J., McFadden, J. P., Larson, D. E., Eriksson, S., Johansson, T., Cully, C. M., Newman, D. N., Goldman, M .V., Roux, A., LeContel, O., Glassmeier, K.-H., and Baumjohann, W.: Observation of double layers in Earth's plasma sheet, Phys. Rev. Lett., 102, 155002, doi:10.1103/PhysRevLett.102.155002, 2009.

Fu, X. R., Lu, Q. M., and Wang, S.: The process of electron acceleration during collisionless magnetic reconnection, Phys. Plasma, 13, 012309, doi:10.1063/1.2164808, 2006.

Fujimoto, K. and Machida, S.: A generation mechanism of electrostatic waves and subsequent electron heating in the plasma sheetlobe boundary region during magnetic reconnection, J. Geophys. Res., 111, A09216, doi:10.1029/2005JA011452, 2006.

Fujimoto, K. and Sydora, R. D.: Whistler waves associated with magnetic reconnection, Geophys. Res. Lett., 35, L19112, doi:10.1029/2008GL035201, 2008.

Guo, J.: Excitation of whistler mode waves in collisionless magnetic reconnection with a particle-in-cell simulation, Plasma Phys. Control. Fusion, 53, 022001, doi:10.1088/07413335/53/2/022001, 2011.

Guo, J. and Li, B.: The electron-ion streaming instabilities driven by drift velocities of the order of electron thermal velocity in a nonmagnetized plasma, Astrophys. Space Sci., 347, 99-107, 2013.

Guo, J.: Electric field with bipolar structure during magnetic reconnection without a guide field, Astrophys. Space Sci., 351, 159163, 2014.

Huang, C., Lu, Q. M., and Wang S.: The mechanisms of electron acceleration in anti-parallel and guide filed magnetic reconnection, Phys. Plasma, 17, 072306, doi:10.1063/1.3457930, 2010.

Hoshino, M., Mukai. T., Terasawa, T., and Shinohara, I.: Suprathermal electron acceleration in magnetic reconnection, J. Geophys. Res., 106, 25979-25997, 2001.

Li, S. Y., Deng, X. H., Zhou, M., Tang, R. X., Liu, K., Kojima, H., and Matsumoto, H.: Statistical study of electrostatic solitary 
waves associated with reconnection: Geotail observations, Adv. Space Res., 43, 394-400, 2009.

Lu, Q. M., Huang, C., Xie, J. L., Wang, R. S., Wu, M. Y., Vaivadds, A., and Wang, S.: Features of separatrix regions in magnetic reconnection: Comparison of $2 \mathrm{D}$ particle-in-cell simulations and Cluster observations, J. Geophys. Res., 115, A11208, doi:10.1029/2010JA015713, 2010.

Lu, Q. M., Lu, S., Huang, C., Wu, M. Y., and Wang S.: Self-reinforcing process of the reconnection electric field in the electron diffusion region and onset of collisionless magnetic reconnection, Plasma Phys. Control. Fusion, 55, 085019, doi:10.1088/0741-3335/55/8/085019, 2013.

Pritchett, P. L.: Relativistic electron production during guide field magnetic reconnection, J. Geophys. Res., 111, A10212, doi:10.1029/2006JA011793, 2006.

Pritchett, P. L. and Coroniti, F. V.: Three-dimensional collisionless magnetic reconnection in the presence of a guide field, J. Geophys. Res., 109, A01220, doi:10.1029/2003JA009999, 2004.

Singh, N.: Computer experiments on the formation and dynamics of electric double layers, Plasma Phys., 22, 1-24, 1980.

Singh, N.: Double-layer formation, Plasma Phys., 24, 639-660, 1982.

Singh, N.: Electron holes as a common feature of double-layerdriven plasma waves, Geophys. Res. Lett., 27, 927-930, 2000.

Singh, N. and Khazanov, I.: Dynamic planar double layers: Filamentary substructures, electron holes and ion heating, Geophys. Res. Lett., 30, 2262, doi:10.1029/2003GL018480, 2003.

Singh, N., Deverapalli, C., and Khazanov, I.: Comparison of electric fields and density structures seen in simulations and satellite observations, Geophys. Res. Lett., 32, L13108, doi:10.1029/2005GL022920, 2005.
Singh, N., Araveti, S., and Wells, E. B.: Mesoscale PIC simulation of double layers and electron holes affecting parallel and transverse accelerations of electrons and ions, J. Geophys. Res., 116, A00K09, doi:10.1029/2010JA016323, 2011.

Vaivads, A., Khotyaintsev, Y., Andre, M., Retinò, A., Buchert, S. C., Rogers, B. N., Decreau, P., Paschmann, G., and Phan, T. D.: Structure of the magnetic reconnection diffusion region from four-spacecraft observations, Phys. Rev. Lett., 93, 105001, doi:10.1103/PhysRevLett.93.105001, 2004.

Wang, R. S., Du, A. M., Nakamura, R., Lu, Q. M., Khotyaintsev, Y. V., Volwerk, M., Zhang, T. L., Kronberg, E. A., Daly, P. W., and Fazakerley, A. N.: Observation of multiple sub-cavities adjacent to single separatrix, Geophys. Res. Lett., 40, 2511-2517, doi:10.1002/grl.50537, 2013.

Wang, R. S., Lu, Q. M., Khotyaintsev, Y. V., Volwerk, M., Du, A. M.,Nakamura, R., Gonzalez, W. D., Sun, X., Baumjohann, W., Li, X., Zhang, T. L., Fazakerley, A. N., Huang, C., and Wu, M. Y.: Observation of double layer in the separatrix region during magnetic reconnection, Geophys. Res. Lett., 41, 4851-4858, doi:10.1002/2014GL061157, 2014

Xiao, F. L., Chen, L. X., Zhou, Q. H., He, H. Y., and Wen, Y. J.: Bounce-averaged acceleration of energetic electrons by whistler mode chorus in the magnetosphere, Chin. Phys. Lett., 24, 294 297, 2007.

Xiao, F. L., Chen, L. X., He, H. Y., and Zhou, Q. H.: Second-order resonant interaction of ring current protons with whister-mode waves, Chin. Phys. Lett., 25, 336-339, 2008.

Xiao, F. L., Zong, Q. G., Pu, Z. Y., Su, Z. P., Cao, J. B., He, J. S., Wang, Y. F., and Zheng, H. N.: Electron acceleration by whistlermode waves around the magnetic null during 3D reconnection, Plasma Phys. Control. Fusion, 52, 052001, doi:10.1088/07413335/52/5/052001, 2010. 